\title{
Influence of nuclear exchange on nonadiabatic electron processes in $\mathrm{H}^{+}+\mathrm{H}_{2}$ collisions
}

\author{
L. F. Errea, ${ }^{1}$ Clara Illescas,${ }^{1}$ A. Macías,${ }^{1}$ L. Méndez, ${ }^{1}$ B. Pons,${ }^{2}$ I. Rabadán, ${ }^{1, a)}$ and \\ A. Riera ${ }^{1}$ \\ ${ }^{1}$ Laboratorio Asociado al CIEMAT de Física Atómica y Molecular en Plasmas de Fusión, Departamento de \\ Química, Universidad Autónoma de Madrid, Madrid-28049, Spain \\ ${ }^{2}$ Centre Lasers Intenses et Applications, Université de Bordeaux-I-UMR 5107 CNRS-CEA, 351 Cours de la \\ Libération, 33405 Talence, France
}

(Received 23 July 2010; accepted 30 October 2010; published online 28 December 2010)

\begin{abstract}
$\mathrm{H}^{+}+\mathrm{H}_{2}$ collisions are studied by means of a semiclassical approach that explicitly accounts for nuclear rearrangement channels in nonadiabatic electron processes. A set of classical trajectories is used to describe the nuclear motion, while the electronic degrees of freedom are treated quantum mechanically in terms of a three-state expansion of the collision wavefunction. We describe electron capture and vibrational excitation, which can also involve nuclear exchange and dissociation, in the $E=2-1000 \mathrm{eV}$ impact energy range. We compare dynamical results obtained with two parametrizations of the potential energy surface of $\mathrm{H}_{3}^{+}$ground electronic state. Total cross sections for $E>10$ $\mathrm{eV}$ agree with previous results using a vibronic close-coupling expansion, and with experimental data for $E<10 \mathrm{eV}$. Additionally, some prototypical features of both nuclear and electron dynamics at low $E$ are discussed. (C) 2010 American Institute of Physics. [doi:10.1063/1.3518417]
\end{abstract}

\section{INTRODUCTION}

Collisions between protons and hydrogen molecules are the natural benchmark for the improvement and implementation of new theoretical approaches in ion-molecule collisions. Particularly interesting is the $E \approx 1-100 \mathrm{eV}$ impact energy range where nuclear exchange $(\mathrm{NE})$ reactions compete with direct nonadiabatic electron processes. If we refer to the impinging proton as $\mathrm{C}^{+}$, while the initial target is identified as $\mathrm{AB}$, the most significant processes that take place from the ground vibrational $(v=0)$ state are:

- Elastic scattering:

$$
\mathrm{C}^{+}+\mathrm{AB}\left(X^{1} \Sigma_{g}^{+}, v=0\right) \rightarrow \mathrm{C}^{+}+\mathrm{AB}\left(X^{1} \Sigma_{g}^{+}, v=0\right) .
$$

- Nonreactive vibrational excitation (NRVE):

$\mathrm{C}^{+}+\mathrm{AB}\left(X^{1} \Sigma_{g}^{+}, v=0\right) \rightarrow \mathrm{C}^{+}+\mathrm{AB}\left(X^{1} \Sigma_{g}^{+}, v\right)$.

- Dissociation:

$\mathrm{C}^{+}+\mathrm{AB}\left(X^{1} \Sigma_{g}^{+}, v=0\right) \rightarrow \mathrm{C}^{+}+\mathrm{A}+\mathrm{B}$.

- Nonreactive electron capture (NREC):

$\mathrm{C}^{+}+\mathrm{AB}\left(X^{1} \Sigma_{g}^{+}, v=0\right) \rightarrow \mathrm{C}+\mathrm{AB}^{+}\left(X^{2} \Sigma_{g}^{+}, v^{\prime}\right)$.

\footnotetext{
a)Electronic mail: ismanuel.rabadan@uam.es.
}

- Dissociative electron capture (DEC):

$$
\begin{aligned}
\mathrm{C}^{+}+\mathrm{AB}\left(X^{1} \Sigma_{g}^{+}, v=0\right) & \rightarrow \mathrm{C}+\mathrm{A}^{+}+\mathrm{B} \\
& \rightarrow \mathrm{C}+\mathrm{B}^{+}+\mathrm{A} .
\end{aligned}
$$

- Nuclear exchange:

$$
\begin{aligned}
\mathrm{C}^{+}+\mathrm{AB}\left(X^{1} \Sigma_{g}^{+}, v=0\right) & \rightarrow \mathrm{A}^{+}+\mathrm{BC}\left(X^{1} \Sigma_{g}^{+}, v=0\right) \\
& \rightarrow \mathrm{B}^{+}+\mathrm{AC}\left(X^{1} \Sigma_{g}^{+}, v=0\right)
\end{aligned}
$$

- Reactive vibrational excitation (RVE):

$$
\begin{aligned}
\mathrm{C}^{+}+\mathrm{AB}\left(X^{1} \Sigma_{g}^{+}, v=0\right) & \rightarrow \mathrm{A}^{+}+\mathrm{BC}\left(X^{1} \Sigma_{g}^{+}, v\right) \\
& \rightarrow \mathrm{B}^{+}+\mathrm{AC}\left(X^{1} \Sigma_{g}^{+}, v\right) .
\end{aligned}
$$

- Reactive electron capture (REC):

$$
\begin{aligned}
\mathrm{C}^{+}+\mathrm{AB}\left(X^{1} \Sigma_{g}^{+}, v=0\right) & \rightarrow \mathrm{A}+\mathrm{BC}^{+}\left(X^{2} \Sigma_{g}^{+}, v^{\prime}\right) \\
& \rightarrow \mathrm{B}+\mathrm{AC}^{+}\left(X^{2} \Sigma_{g}^{+}, v^{\prime}\right)
\end{aligned}
$$

Some of these reactions, particularly the electron capture ones, are relevant in first stars formation, since they are the dominant $\mathrm{H}_{2}$ destruction processes, and $\mathrm{H}_{2}$ is the main coolant of the primordial gas (see Refs. 1 and 2). 
Among the theoretical studies of $\mathrm{H}^{+}+\mathrm{H}_{2}$ collisions, only the electron nuclear dynamics (END) ${ }^{3}$ and trajectory surface hopping (TSH $)^{4}$ calculations have simultaneously considered the nonreactive [Eqs. (1)-(5)] and reactive [Eqs. (6)-(8)] processes. Collisions involving the isotopically modified species $\mathrm{DH}_{2}^{+}$have also been studied at $1.5<E<2.5 \mathrm{eV}$ using closecoupling, ${ }^{5}$ wave-packet, ${ }^{6,7}$ and TSH methods. ${ }^{8}$ Baer et al. ${ }^{9}$ provided theoretical cross sections for single capture [Eq. (4)] and vibrational excitation (VE) [Eq. (2)], obtained at $E=30$ $\mathrm{eV}$ using the infinite order sudden approximation (IOSA) with diatomics-in-molecules (DIM) potentials. Ab initio calculations of Saieswari and Kumar ${ }^{10}$ yielded results in agreement with those of Baer et al. Krstic ${ }^{11}$ and Krstić and Janev ${ }^{12}$ also employed IOSA at $E<15 \mathrm{eV}$, and further considered dissociative processes. More recently, Amaran and $\mathrm{Kumar}^{13}$ have evaluated differential cross sections for electron capture and vibrational excitation at energies between 4.67 and $10 \mathrm{eV}$.

At high energies, Illescas and Riera ${ }^{14}$ performed a classical mechanics study of ionization and electron capture [Eq. (4)] in $\mathrm{H}^{+}+\mathrm{H}_{2}$ collisions. Elizaga et al. ${ }^{15}$ also investigated electron capture in the same collisions using an eikonal treatment with the sudden approximation to describe target vibration and rotation. Although the sudden approximation is more accurate than the simple Franck-Condon (FC) approximation, it was found that it severely underestimates the capture cross section measured in the ion-beam-gas-cell ${ }^{16,17}$ and crossed-beams experiments ${ }^{18}$ for $E<200 \mathrm{eV}$. The origin of this failure was traced back ${ }^{19}$ to the inability of the sudden approximation to represent the two-step quasiresonant mechanism, responsible for capture at low energies. The two-step mechansim, proposed by Niedner et al. ${ }^{20}{ }^{20}$ involves the vibrational excitation from the $\mathrm{H}_{2}$ ground vibrational state $(v=0)$ to excited ones $(v=3,4)$, followed by the quasiresonant electronic nonadiabatic transition to the capture state dissociating into $\mathrm{H}(1 s)+\mathrm{H}_{2}^{+}\left(X^{2} \Sigma_{g}^{+}, v^{\prime}=0\right)$. Accordingly, we carried out a calculation that did not employ the sudden approximation for vibration; we reported cross sections for electron capture and vibrational excitation (including dissociative processes), obtained from quantal and semiclassical vibronic closecoupling (VCC) expansions of the dynamical wavefunction with the sudden approximation for target rotation..$^{21}$ The VCC results, which employed $a b$ initio electronic wavefunctions, nicely coincide with previous IOSA calculations at $E=30$ $\mathrm{eV},{ }^{9}$ which used DIM electronic wavefunctions ${ }^{22}$ and a small vibronic basis set, but they do not agree with END (Ref. 3) and TSH (Ref. 4) cross sections. Furthermore, the VCC single capture cross section presents a maximum near $E=30$ $\mathrm{eV}$, which is not present in the recommended data of Phelps. ${ }^{23}$ No experimental data have been reported at these energies.

We have recently developed a theoretical method, ${ }^{24}$ called classical trajectory-diatomics in molecules (CTDIM), aiming at checking the reliability of the approximations introduced in previous VCC (and related IOSA) calculations at low energies. In particular, as explained in Ref. 24, CTDIM does not use the sudden approximation for rotation; it explicitly includes nuclear exchange channels and does not limit the wavefunction expansion to the two lowest $\mathrm{H}_{3}^{+}$adiabatic states. CTDIM is semiclassical: it merges classical mechanics to describe the motion of the nuclei and a quantum representation of the electron dynamics. Concretely, the motion of both projectile and target nuclei under the influence of the ground potential energy surface (PES) of $\mathrm{H}_{3}^{+}$is obtained by means of statistical classical trajectory Monte Carlo (CTMC) ${ }^{25,26}$ simulations. Then, for each nuclear trajectory, the electron dynamics is described by the semiclassical Schrödinger equation which is solved by using a three-state DIM expansion.

In Ref. 24, we reported electron capture and vibrational excitation cross sections obtained from preliminary CTDIM calculations using the parametrized $\mathrm{H}_{3}^{+}$PES of Giese and Gentry $^{27}\left(V_{\mathrm{GG}}\right)$. These cross sections were found in acceptable agreement with their VCC counterparts for $E \geq 20 \mathrm{eV}$, and the maximum of the VCC capture cross section around $30 \mathrm{eV}$ was attributed to the lack of explicit nuclear exchange channels in the VCC expansion. However, the reliability of our preliminary CTDIM calculations ${ }^{24}$ at low $E$ might be questioned since the parametrized PES $V_{\mathrm{GG}}$ is known to be inaccurate for large diatomic bond distances, such as those involved in nuclear exchange and dissociation processes. ${ }^{27} \mathrm{In}$ this respect, Ichihara et al. ${ }^{4}$ have noted that, in the framework of TSH calculations, $\mathrm{H}^{+}+\mathrm{H}_{2}$ inelastic cross sections are indeed sensitive, within 20\%, to the accuracy of the PES employed. Accordingly, we present in this contribution CTDIM calculations that employ the analytical parametrization of Velilla et $a .^{28}\left(V_{\mathrm{VLA}}\right)$, based on large-scale ab initio calculations. We gauge the relation between the accuracy of the PES and calculated cross sections by direct comparison of both nuclear and electron dynamics using $V_{\mathrm{GG}}$ and $V_{\mathrm{VLA}}$. All calculations use nuclear statistics larger than those employed in Ref. 24, where the relatively small number of nuclear trajectories considered led to spurious oscillations in the cross sections. Finally, and in contrast to what was presented in Ref. 24 , we analyze in detail the nuclear and electronic dynamics leading to nonadiabatic processes with and without nuclear rearrangement. In this respect, the inclusion of reactive channels allows us to consider a wide range of impact energies, $2<E<1000 \mathrm{eV}$, where we discuss the processes responsible for the shape of the electron capture cross section, and, in particular, the relative importance of reactive and nonreactive electron capture processes.

Our paper is organized as follows: the CTMC description of nuclear motion and the DIM representation of electron dynamics are presented in Sec. II; in Sec. III, we picture the nuclear dynamics associated to the main reaction paths, and present the electron capture and excitation cross sections obtained in CTDIM calculations. In Sec. III we analyze the collision mechanisms by displaying some representative collision histories, where the correlation between nuclear motion and electron dynamics clearly shows up. In Sec. IV, we present our conclusions and perspectives. Atomic units are used throughout the paper unless otherwise stated.

\section{CTDIM METHOD}

\section{A. Nuclear dynamics}

At the collision energies considered in this work, the rotational period of the $\mathrm{H}_{2}$ molecule is large compared to the 


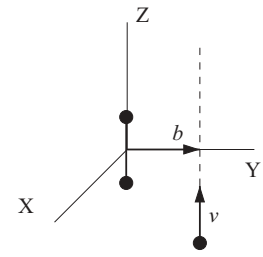

T I

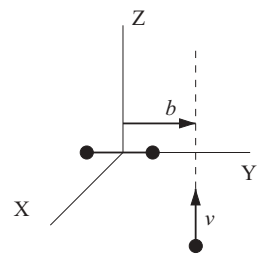

T II

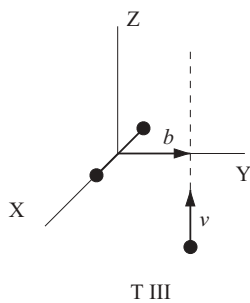

T III
FIG. 1. Different orientations of the initial $\mathrm{H}_{2}$ diatom with respect to the impinging $\mathrm{H}^{+}$ion, corresponding to the three types of trajectory employed to evaluate orientation averaged probabilities and cross sections.

collision characteristic time, and we have accordingly assumed that initially the $\mathrm{H}_{2}$ target does not rotate. This simplification could have been avoided, but this would have seriously enlarged the number of nuclear and electronic equations to be solved, without substantial improvement of the dynamical results.

\section{Target-projectile relative orientations}

The nuclear positions of the $\mathrm{C}^{+}+\mathrm{AB}$ system are defined by the Jacobi coordinates $\{\boldsymbol{\rho}, \mathbf{R}\}$, where $\boldsymbol{\rho}$ corresponds to the internuclear vector of the diatom and $\mathbf{R}$ stands for the vector of the third center with respect to the center of mass of the diatom. In the laboratory reference frame, the projectile motion is initially described by an impact parameter $\mathbf{b}$ in the $Y$-axis direction, and an initial velocity $\mathbf{v}$ in the $Z$-axis direction. For the calculation of probabilities and cross sections averaged over different relative target-projectile orientations, we consider, as in Ref. 29, the three different types of trajectories illustrated in Fig. 1. These trajectories differ in the alignment of the target molecule along one of the laboratory-fixed axis: the first one, labeled TI, corresponds to $\hat{\boldsymbol{\rho}} \| \hat{\mathbf{Z}}$, while TII and TIII correspond to $\hat{\boldsymbol{\rho}} \| \hat{\mathbf{Y}}$, and $\hat{\boldsymbol{\rho}} \| \hat{\mathbf{X}}$, respectively.

\section{Classical description of the initial vibrational state}

The initial condition for the $\mathrm{AB}$ diatom corresponds to the vibroelectronic eigenstate $\psi_{i} \chi_{i, v}$, where $\psi_{i}$ is the ground electronic $\mathrm{H}_{2}\left(X^{1} \Sigma_{g}^{+}\right)$state, and $\chi_{i, v=0}(\rho)$ is the ground $\mathrm{H}_{2}$ vibrational function associated to this electronic state. $\chi_{i, v=0}$, as well as the excited and continuum $\chi_{i, v}$ vibrational states, can be quite accurately reproduced using the Morse potential

$$
V(\rho)=D\left[e^{-2 \alpha\left(\rho-\rho_{0}\right)}-2 e^{-\alpha\left(\rho-\rho_{0}\right)}\right]
$$

as an approximation to the $\mathrm{H}_{2}$ ground electronic potential, where $D=0.17456 E_{h}$ is the potential depth, $\rho_{0}=1.4022 a_{0}$ is the $\mathrm{H}_{2}$ equilibrium distance, and $\alpha=1.0271\left(a_{0}\right)^{-1} . V(\rho)$ yields the eigenvalues $E_{v}$ for the bound vibrational states $\chi_{i, v}$,

$$
E_{v}=\omega\left[\left(v+\frac{1}{2}\right)-\frac{\omega}{4 D}\left(v+\frac{1}{2}\right)^{2}\right],
$$

with $\omega \approx 0.02 E_{h}$, so that the energy $E_{0}$ of the ground vibrational state is approximately $0.01 E_{h}$ (referred to as the bottom of the potential well).

Some semiclassical methods, such as distribution (among quantum states) of exact classical energy transfer (DECENT) (Refs. 27 and 30) or END (Ref. 3), employ a simplified initial condition where the target nuclei are located at the equilibrium distance $\rho_{0}$. Our description of vibration is completely different: it is based on a statistical (Monte Carlo) representation that mimics the quantum $\chi_{i, v=0}(\rho)$ initial state. In such a representation, the most common procedure consists in distributing, in the $\left(\rho, p_{\rho}\right)$ phase space, noninteracting trajectories with energy $E_{0}=$ $p_{\rho}^{2} /(2 \mu)+V$. By doing so, one builds a so-called microcanonical distribution, $\varrho\left(E_{0} ; \rho, p_{\rho}\right)$, which spatial density, $\varrho\left(E_{0} ; \rho\right)=\int \varrho\left(E_{0} ; \rho, p_{\rho}\right) d p_{\rho}$, presents-in the case of the Morse potential-the analytical form ${ }^{31}$

$$
\varrho\left(E_{0} ; \rho\right)=\frac{\alpha}{\pi}\left[\frac{V(\rho)}{E_{0}-D}-1\right]^{-1 / 2} .
$$

As shown in Fig. 2, the microcanonical description is clearly unsatisfactory since $\varrho\left(E_{0} ; \rho\right)$ diverges at both inner $\rho_{\min }$ and outer $\rho_{\max }$ classical turning points of $V(\rho)$, because of the reduced (1D) dimensionality of vibrational motion. Moreover, the microcanonical description has been shown to lead to inaccurate cross sections at high impact energies, where the sudden approximation for vibration holds. ${ }^{31}$

In the present work, we construct an improved initial distribution based on the use of the phase space Wigner distribution $^{32}$

$$
W\left(\rho, p_{\rho}\right)=\frac{1}{2 \pi} \int d \eta e^{-i p_{\rho} \eta} \chi_{i, 0}^{*}\left(\rho-\frac{\eta}{2}\right) \chi_{i, 0}\left(\rho+\frac{\eta}{2}\right),
$$

which is known to reproduce exactly the quantum densities in both configuration and momentum spaces. The classical Wigner energy distribution

$$
\mathcal{E}_{W}(\epsilon)=\int W\left(\rho, p_{\rho}\right) \delta\left(p^{2} / 2 \mu+V-\epsilon\right) d \rho d p_{\rho}
$$

spreads from 0 to $\infty$, as shown in Fig. 2(a), and verifies $\left\langle\mathcal{E}_{W}(\epsilon)\right\rangle=E_{0}$ (Refs. 33 and 34). Within classical calculations, $W\left(\rho, p_{\rho}\right)$ can be reconstructed in terms of a functional integral over microcanonical distributions $\varrho\left(\epsilon ; \rho, p_{\rho}\right)$ weighted by $\mathcal{E}_{W}(\epsilon)$. But one has first to truncate the spread of the Wigner energy distribution according to the partition of the energy scale previously detailed in Ref. 31. In our case, this leads to the truncated distribution $\mathcal{E}_{W t}(\epsilon)$ :

$$
\mathcal{E}_{W t}(\epsilon)=\left\{\begin{array}{lll}
0 & \text { if } \quad E_{\min }>\epsilon \geq E_{0}^{+} \\
\mathcal{E}_{W}(\epsilon) / N_{W} & \text { if } \quad E_{\min } \leq \epsilon<E_{0}^{+}
\end{array}\right.
$$

where $E_{0}^{+} \approx 0.02 E_{h}$ is the upper bound of the classical energy bin associated to the ground $v=0$ vibrational state. ${ }^{31}$ To calculate $\mathcal{E}_{W t}(\epsilon)$, the lower bound $E_{\min }$ is not set equal to 0 , and $N_{W}$ is introduced in order to comply with both $\int_{E_{\min }}^{E^{+}} \mathcal{E}_{W t}(\epsilon) d \epsilon=1$ and $\int_{E_{\min }}^{E^{+}} \mathcal{E}_{W t}(\epsilon) \epsilon d \epsilon=0.01 E_{h}$. In practice, $E_{\min }=0.0041 E_{h}$ and $N_{W}=0.5414$. $\mathcal{E}_{W t}(\epsilon)$ is compared to $\mathcal{E}_{W}(\epsilon)$ in Fig. 2(a). Finally, our improved initial condition consists of a discrete representation of

$$
\varrho_{W t}\left(\rho, p_{\rho}\right)=\int_{E_{\min }}^{E_{0}^{+}} \mathcal{E}_{W t}(\epsilon) \varrho\left(\epsilon ; \rho, p_{\rho}\right) d \epsilon
$$

in terms of five microcanonical distributions $\varrho\left(\epsilon ; \rho, p_{\rho}\right)$, totaling 100 trajectories. The associated spatial density is displayed in Fig. 2(b), where one can note that it is free from 

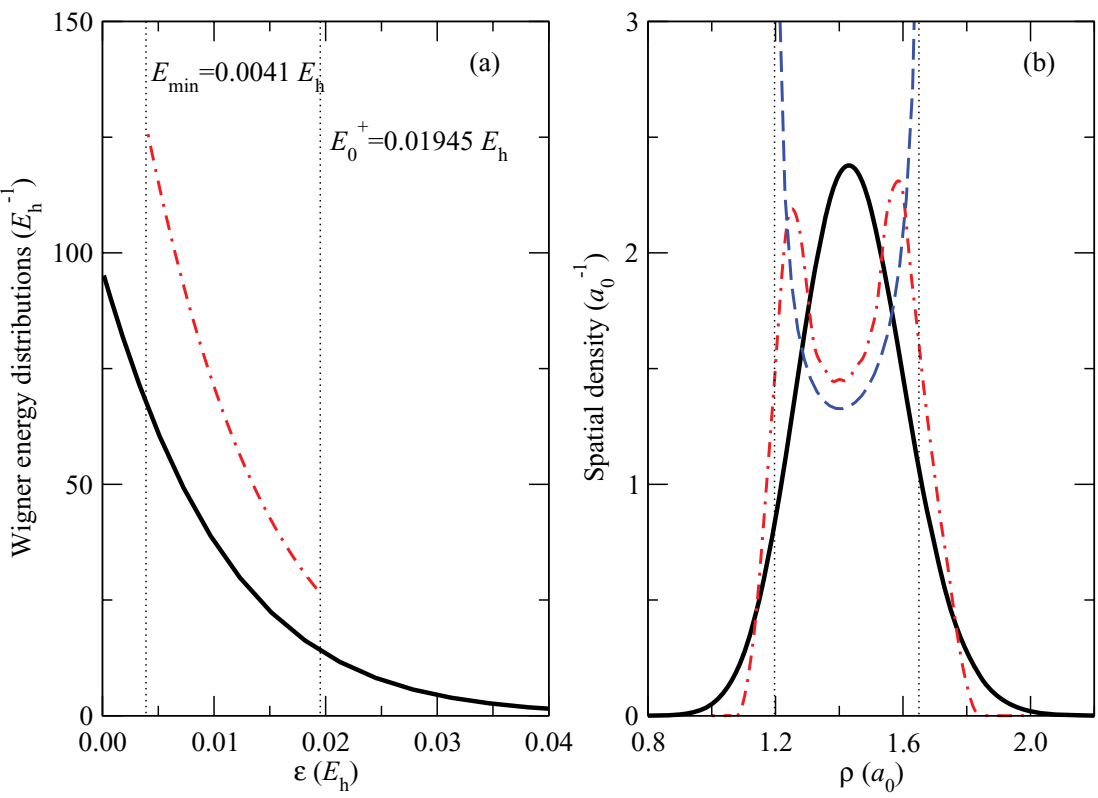

FIG. 2. (a) Integral (solid line) and truncated (dotted-dashed line) classical Wigner energy distributions corresponding to Eqs. (13) and (14), respectively. (b) Quantal density of the $v=0$ vibrational state of fundamental $\mathrm{H}_{2}$ (solid line), compared to single microcanonical (dashed line) and truncated Wigner (dotted-dashed) descriptions.

divergences, and it extends over a range of $\rho$ values larger than that of the corresponding single microcanonical distribution. To bring the classical and quantal distributions even closer, i.e., filling the dip about $\rho=\rho_{0}$ and further enlarging the ranges of $\varrho_{W t}(\rho)$, is not feasible insomuch as it would necessitate to extend $\mathcal{E}_{W t}(\epsilon)$ beyond the limits imposed by the classical partition of the negative energy scale. Taking into account the three target-projectile relative orientations considered in Fig. 1, we run $N=300$ different nuclear trajectories, labeled as $\kappa$, for each pair of initial relative velocity, $v$, and impact parameter $b$.

\section{Nuclear evolution}

The evolution of the noninteracting nuclear trajectories is determined by the Hamilton equations

$$
\dot{\mathbf{R}}_{j}=\mathbf{P}_{j}, \quad \dot{\mathbf{P}}_{j}=-\nabla V_{\mathrm{PES}},
$$

where $V_{\mathrm{PES}}$ is the PES of the $\mathrm{H}_{3}^{+}$ground electronic state, and $\mathbf{R}_{j}$ and $\mathbf{P}_{j}$, with $j=\mathrm{A}, \mathrm{B}$, or $\mathrm{C}$, correspond to the position and momentum nuclear vectors. As mentioned in the Introduction, we have selected the parametrization $V_{\mathrm{GG}}$ of Ref. 27 , also employed in the calculation of vibrational excitation cross sections [Eq. (2)] of Ref. 30. As in conventional DIM treatments, $V_{\mathrm{GG}}$ consists of a sum over diatomic potential functions similar to Eq. (9), in which the width, depth, and position of minimum is allowed to vary from the values of the unperturbed $\mathrm{H}_{2}$. Additionally, the long-range chargeinduced dipole and quadrupole contributions to the $\mathrm{H}^{+}+\mathrm{H}_{2}$ potential employ quadrupole moment and polarizability functions fitted to cubic polynomia of $\rho$ near the equilibrium position. Therefore, $V_{\mathrm{GG}}$ is inaccurate at $\rho \gg \rho_{0}$. Furthermore, $V_{\mathrm{GG}}$ has been originally designed to describe $\mathrm{H}^{+}+\mathrm{H}_{2}$ collisions without rearrangement; in the case of nuclear exchange and dissociation processes, $\rho$ and $\mathbf{R}$ are redefined during the collision. This is not necessary when using the PES of Velilla et al. ${ }^{28} V_{\mathrm{VLA}}$. This PES is constructed on DIM grounds, but all long-range terms are included in the diagonal elements of the DIM matrix, yielding a global potential function symmetric under any permutation of the three identical nuclei. The final PES, defined in terms of bond coordinates, instead of Jacobi ones, is therefore well suited to dynamical studies of reactive and nonreactive processes in $\mathrm{H}^{+}+\mathrm{H}_{2}$ collisions. Moreover, the semianalytical form of all long-range terms involve fitting procedures to $a b$ initio data issued from state-of-theart quantum chemistry calculations, beyond the description of Ref. 27. In the present work, we have used the $V_{\mathrm{VLA}}$ PES in order to gauge the impact of the accuracy of the PES on the dynamical results.

The Cartesian coordinates of $\mathbf{R}_{j}(t)$, with $j=\mathrm{A}, \mathrm{B}$, and $\mathrm{C}$, are regularly recorded along the integration of the Hamilton equations since they are used as input data to the subsequent treatment of electronic transitions. In practice, the nuclear equations are integrated up to a time $t=t_{\max }$ where at least two of the three diatomic distances reach values larger than $100 a_{0}$; all dynamically allowed nuclear and electronic transitions have taken place within the range $t<t_{\max }$.

Obviously, resonances are not obtained in the classical treatment of the nuclear dynamics. However, as it has been shown in the calculation of Refs. 6 and 7, even at the lowest energies of our calculation, the resonant structures are smoothed out when adding up the contributions of the different partial waves, and are practically unnoticed in the total cross sections.

\section{Identification of final reaction channels}

For a given nuclear trajectory $\kappa$, we distinguish between dissociative and nondissociative paths at time $t_{\max }$ as follows: we register the two larger diatomic distances, say $R_{\mathrm{AC}}$ 
and $R_{\mathrm{BC}}$, and calculate the total diatomic energy $E_{\text {diatom }}$ $=p_{\rho}^{2} /(2 \mu)+V(\rho)$ associated to the remaining diatom, $\mathrm{AB}$, with $\rho=R_{\mathrm{AB}}$ and $p_{\rho}$ its conjugate momentum. The trajectory $\kappa$ belongs to a dissociative channel, that we identify with the label $J_{\kappa}=4$, if $E_{\text {diatom }}>0$ (recall that $\lim _{\rho \rightarrow \infty} V(\rho)=0$ according to Eq. (9)). Otherwise, $E_{\text {diatom }}<0$ and the diatom $\mathrm{AB}$ is bound at the end of a nonreactive process, and we assign $J_{\kappa}=1$. Obviously, nuclear exchange can occur at $t<t_{\max }$, leading to the formation of bound diatoms $\mathrm{AC}$ and $\mathrm{BC}$, respectively marked with $J_{\kappa}=2$ and 3 .

Within nondissociative processes $\left(J_{\kappa}=1,2\right.$, and 3 ), we can resolve the vibrational state reached in the respective bound diatom $(\mathrm{AB}, \mathrm{AC}, \mathrm{BC})$ by defining the classical vibrational energy as $E_{\text {vib }}=E_{\text {diatom }}-E_{\text {rot }}$, where $E_{\text {rot }}$ is the rotational energy. There is no unique way to define $E_{\text {rot }}$. In this work, we have chosen to integrate to longer times, from $t_{\max }$ up to $t_{\max }+5000 \hbar / E_{h}$, after a bound diatom is formed in order to define a mean rotational energy as

$$
E_{\mathrm{rot}}=\left\langle\frac{L^{2}}{2 \mu \rho^{2}}\right\rangle
$$

where $\mathbf{L}$ is the angular momentum $\boldsymbol{\rho} \times \mathbf{p}_{\rho}$, which is conserved throughout the asymptotic region. An alternative to the previous definition is $E_{\text {rot }}=L^{2} /\left(2 \mu\langle\rho\rangle^{2}\right)$; in practice, both definitions lead to almost identical rotational energy transfers. Once $E_{\text {rot }}$ is determined, the classical vibrational level $v_{\kappa}$ reached at the end of the nuclear trajectory $\kappa$ is determined by identifying the classical energy-bin $\left[E_{v}^{-}, E_{v}^{+}\left[\right.\right.$in which $E_{\mathrm{vib}}$ lies. We have not considered state-selective rotational processes, which means that in our calculation the elastic reaction (1) includes target rotational excitation.

\section{B. Electron dynamics}

Once the time-dependence of the nuclear coordinates along the trajectory $\kappa,\left\{\mathbf{R}_{j}(t)\right\}$, is known, the electron dynamics is quantum mechanically described by the semiclassical equation

$$
\left[H_{\mathrm{el}}-i \frac{\partial}{\partial t}\right] \Psi\left(\kappa, v, b ;\left\{\mathbf{r}_{1,2}\right\},\left\{\mathbf{R}_{j}(t)\right\}\right)=0,
$$

where $H_{\mathrm{el}}$ is the clamped-nuclei Born-Oppenheimer Hamiltonian and $\Psi\left(\kappa, v, b ;\left\{\mathbf{r}_{1,2}\right\},\left\{\mathbf{R}_{j}(t)\right\}\right)$, the two-electron total wavefunction for nuclear trajectory $\kappa$ and given values of $v$ and $b$. This equation is solved by expanding $\Psi$ in terms of three (diabatic) DIM states, which yield not only energies but also nonadiabatic couplings in good agreement with their $a b$ initio counterparts, ${ }^{35}$ even in the region of the wellknown conical intersection between the two lowest $\mathrm{H}_{3}^{+} \mathrm{PESs}$ at $R \rightarrow \infty, \rho \approx 2.5 a_{0}$. The expansion has the form

$$
\begin{aligned}
& \Psi\left(\kappa, v, b ;\left\{\mathbf{r}_{1,2}\right\},\left\{\mathbf{R}_{j}(t)\right\}\right) \\
& =\sum_{k=1}^{3} c_{k}(\kappa, v, b ; t) \psi_{k}\left(\left\{\mathbf{r}_{1,2}\right\},\left\{\mathbf{R}_{j}(t)\right\}\right) e^{-i \int_{t_{\mathrm{in}}}^{t} h_{k k}\left(t^{\prime}\right) d t^{\prime}},
\end{aligned}
$$

with $t_{\text {in }}$, the initial time such that $v t_{\text {in }}=-50 a_{0}$, and $h_{k k}$ $=\left\langle\psi_{k}\left|H_{\mathrm{el}}\right| \psi_{k}\right\rangle \cdot \psi_{1}$ describes the channel $\mathrm{C}^{+}+\mathrm{AB}$ while $\psi_{2}$ and $\psi_{3}$, respectively, represent $\mathrm{B}^{+}+\mathrm{AC}$ and $\mathrm{A}^{+}+\mathrm{BC}$. According to the valence-bond character of these DIM states, they can alternatively be interpreted as $\mathrm{C}^{+}+\mathrm{AB}, \mathrm{C}+\mathrm{AB}^{+}\left(\sigma_{g}\right)$ and $\mathrm{C}+\mathrm{AB}^{+}\left(\sigma_{u}\right)$, provided that symmetric and antisymmetric linear combinations of $\psi_{2}$ and $\psi_{3}$ are carried out. The great advantage of the former interpretation is that, as explained below, the definition of electron capture is straightforward when nuclear rearrangement takes place.

Substitution of Eq. (19) into Eq. (18) leads to the system of coupled differential equations for the amplitudes $c_{k}$,

$$
\begin{aligned}
& i \frac{\partial c_{k}(\kappa, v, b ; t)}{\partial t} \\
& \quad=\sum_{l \neq k} c_{l}(\kappa, v, b ; t) h_{k l}(t) e^{-i \int_{t_{\mathrm{in}}}^{t}\left[h_{j j}\left(t^{\prime}\right)-h_{k k}\left(t^{\prime}\right)\right] d t^{\prime}},
\end{aligned}
$$

where $h_{k l}$ are nondiagonal matrix elements of $H_{\mathrm{el}}$ in the DIM set. This system is numerically solved up to $t=t_{\text {max }}$ subject to the initial conditions $c_{l}\left(t_{\text {in }}\right)=\delta_{l 1} e^{-i h_{11}\left(t_{\text {in }}\right)}$. It must be noted that in the DIM representation, derivatives with respect to the internuclear distance $R$ and the Coriolis couplings vanish. Given that the electronic states are assumed to be orthonormal, a small part of the coupling is neglected ${ }^{35}$ in accordance with the impossibility of constructing strict diabatic states in a three-center system (see, e.g., Ref. 36).

The total cross section for single electron capture (SEC) into the nuclear channel $J$ is given by

$$
\sigma_{J}^{\mathrm{SEC}}(v)=\frac{2 \pi}{N} \sum_{\kappa=1}^{N} \int_{0}^{\infty}\left(1-\left|c_{J}\left(\kappa, v, b ; t_{\max }\right)\right|^{2}\right) \delta_{J J_{\kappa}} b d b
$$

where $J=1$ corresponds to the case of NREC [reaction (4)], while $J=2$ and 3 correspond to REC [reactions (8)]. Since simultaneous nuclear exchange and dissociation is unlikely, we consider DEC [reaction (5)] as an extension of NREC, and compute this cross section according to Eq. (21) with $c_{4}=c_{1}$. The total SEC cross section is obviously defined as

$$
\sigma^{\mathrm{SEC}}(v)=\sum_{J=1}^{4} \sigma_{J}^{\mathrm{SEC}}(v)
$$

It is worth noting that we do not provide vibrationally resolved SEC cross sections as the nuclear dynamics have been carried out under the ground $\mathrm{H}_{3}^{+} \mathrm{PES}$, which asymptotically correlates to $\mathrm{H}_{2}$. The FC approximation could have been applied to obtain the populations of the vibrational states of $\mathrm{H}_{2}^{+}$, populated through SEC. Nevertheless, given the limitations of the FC approximation, only valid at high energies (see Ref. 19), we provide total SEC cross sections regardless of the final $\chi_{v^{\prime}}$ state.

The total cross section for VE into the final $v \neq 0$ state of $\mathrm{H}_{2}$ and nuclear channel $J$ is calculated as

$$
\sigma_{J}^{\mathrm{VE}, v}(v)=\frac{2 \pi}{N} \sum_{\kappa=1}^{N} \int_{0}^{\infty}\left|c_{J}\left(\kappa, v, b ; t_{\max }\right)\right|^{2} \delta_{J J_{\kappa}} \delta_{v v_{\kappa}} b d b,
$$

where, as for SEC, $J=1$ corresponds to NRVE [reaction (2)], whereas $J=2$ and 3 correspond to RVE [reaction (7)]. The dissociation [reaction (3)] is computed according to 
Eq. (23) with $J=4$ and $c_{4}=c_{1}$. The total cross section for $\mathrm{VE}$ into the final $v \neq 0$ state is given by

$$
\sigma^{\mathrm{VE}, v}(v)=\sum_{J=1}^{4} \sigma_{J}^{\mathrm{VE}, v}(v)
$$

Finally, we define the elastic cross section $[J=1$, reaction (1)] as

$$
\begin{aligned}
\sigma_{J=1}^{\mathrm{El}}(v)= & \frac{2 \pi}{N} \sum_{\kappa=1}^{N} \int_{0}^{\infty} \mid c_{1}\left(\kappa, v, b ; t_{\max }\right) e^{-i \int_{t_{\mathrm{in}}}^{t_{\max }}\left(h_{11}\left(t^{\prime}\right)-h_{11}\left(t_{\max }\right)\right) d t^{\prime}} \\
& -\left.1\right|^{2} \delta_{1 J_{\kappa}} \delta_{0 v_{\kappa}} b d b
\end{aligned}
$$

while for the cases of nuclear exchange $J=2$ and 3 [reactions (6)] it is

$\sigma_{J \neq 1}^{\mathrm{El}}(v)=\frac{2 \pi}{N} \sum_{\kappa=1}^{N} \int_{0}^{\infty}\left|c_{J}\left(\kappa, v, b ; t_{\max }\right)\right|^{2} \delta_{J J_{\kappa}} \delta_{0 v_{\kappa}} b d b$.

\section{RESULTS}

\section{A. Nuclear dynamics}

In this section, we illustrate the nuclear dynamics of the $\mathrm{C}^{+}+\mathrm{AB}$ system for prototypical trajectories consisting of a target initially located at the bottom of the $\mathrm{H}_{2}$ potential well and the $\mathrm{H}^{+}$impinging ion with energy $E=10 \mathrm{eV}(v \approx 0.02$ $\left.a_{0} E_{h} / \hbar\right)$ and impact parameter $b$. Furthermore, these illustrations correspond to the projectile-target relative orientation TII (see Fig. 1), which is the most amenable to NE and dissociation processes for relatively large $b$, up to $b \approx 1.5 a_{0}$.

As an illustration of the influence in the nuclear trajectories of the ground state PES, we compare in Fig. 3 several parametrizations and computations of the $\mathrm{H}_{3}^{+}$potential along specific trajectories, with $b=1$ and $3 a_{0}$, leading to NE and nonreactive reactions, respectively. The parametrization $V_{\mathrm{GG}}$ is used to obtain the Cartesian coordinates $\mathbf{R}_{j}(v, b ; t)$ of the three nuclei along the trajectories. Afterward these coordinates are used to compute the potential $V_{\mathrm{VLA}}$ and the corresponding $a b$ initio potential, $V_{\mathrm{FCI}}$, issued from a full configuration interaction (FCI) calculation. ${ }^{35}$ For both impact parameters, $b=1$ and $3 a_{0}, V_{\mathrm{VLA}}$ and $V_{\mathrm{FCI}}$ strikingly agree throughout the nuclear path, while $V_{\mathrm{GG}}$ slightly deviates from $V_{\mathrm{FCI}}$, especially for the NE trajectory with $b=1 a_{0}$ [Fig. 3(a)], near the transition state; $V_{\mathrm{GG}}$ is slightly more repulsive for $|v t| \lesssim 5 a_{0}$ in both inner $(v t<0)$ and outer $(v t>0)$ regions. Although these deviations are small, they can significantly influence the subsequent evolution of nuclear coordinates and, accordingly, modify both the nuclear energy transfers and the electron transition probabilities at low impact energies.

The effect of the various PES parametrizations on final electron probabilities shall be examined in the Sec. III B. Here we focus on the nuclear energy transfers obtained by means of the $V_{\mathrm{GG}}$ and $V_{\mathrm{VLA}}$ calculations; Fig. 4 includes the translational $\Delta E_{\text {trans }}$, vibrational $\Delta E_{\mathrm{vib}}$ and rotational $\Delta E_{\mathrm{rot}}$ energy transfers in the range of impact parameters where $V_{\mathrm{GG}}$-CTDIM calculations lead to either NE or nonreactive processes. The $V_{\mathrm{GG}}-\mathrm{CTDIM}$ and $V_{\mathrm{VLA}}$-CTDIM calculations

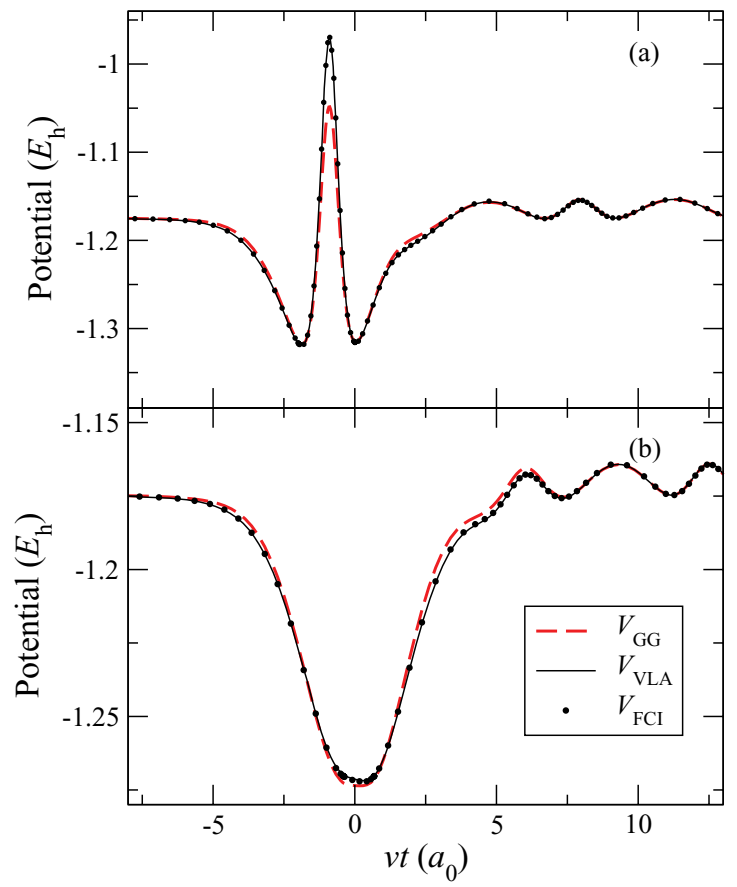

FIG. 3. Comparison of potentials $V_{\mathrm{GG}}, V_{\mathrm{VLA}}$ and $V_{\mathrm{FCI}}$ along (a) a reactive (impact parameter $\left.b=1 a_{0}\right)$ and (b) a nonreactive $\left(b=3 a_{0}\right)$ trajectory with relative projectile-target orientation TII (see Fig. 1) and $E=10 \mathrm{eV}$. The trajectories have been obtained by means of a CTDIM calculation with the target initially located at its equilibrium position.

yield similar energy transfers in the whole range of impact parameters; this behavior holds for all impact energies $E$, even in the $E<10 \mathrm{eV}$ region. As expected, in both the NE and elastic regions, bounded by $0.8 a_{0} \leq b \leq 1.5 a_{0}$ and $b \geq 1.75$ $a_{0}$, respectively, inner- $b$ collisions favor energy transfer to the internal degrees of freedom of the final bounded diatom, while large- $b$ attacks enhance the translational motion of the diatom. Such dynamics can be intuitively understood by means of Figs. 5(d) and 5(e) for NE, and 5(f) and 5(g) for nonreactive collisions.

In Fig. 5(b), we illustrate a dissociative process for a collision with $b=0.3 a_{0}$, and report in Fig. 5(c) the temporal evolution of the atomic kinetic energies. As it could be intuitively expected, collisions with $b \leq \rho_{0} / 2$ inevitably lead to dissociation of the initial diatom AB [see Fig. 5(a)]. Dissociation is a sudden process which occurs as the projectile crosses the internuclear segment. Nevertheless, the kinetic energies reach their asymptotic values only when all centers have receded from each other at $v t \approx 10 a_{0}$, where all long-range interactions are so weak that they do not significantly influence the dynamics anymore [see Fig. 5(c)]. This corresponds to a time delay that roughly behaves as $1 / v$; i.e., some femtoseconds, at $E=10 \mathrm{eV}$. The same delay is necessary to stabilize the internal energy of the diatom in the elastic and NE processes [see Figs. 5(e) and 5(g)] because of the interactions with the escaping atom. Besides the expected dissociation paths at $b \leq \rho_{0} / 2$, one can note in Fig. 5(a) that further dissociation processes occur around $b=1.6 a_{0}$, between the NE and elastic $b$-regions. Figure 4 indicates that these processes involve large energy transfers, which preclude the stabilization of any diatom. 


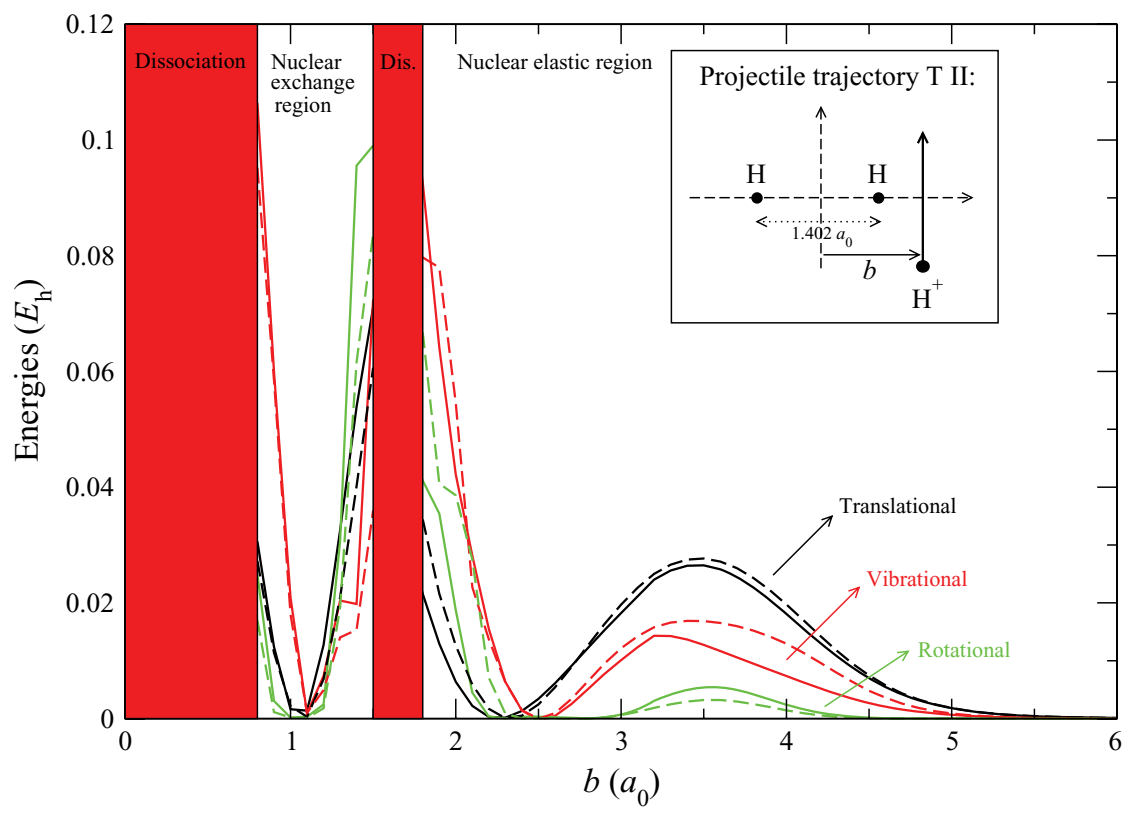

FIG. 4. Translational, vibrational, and rotational energy transfers as functions of the impact parameter $b$, in $10 \mathrm{eV} \mathrm{H}^{+}+\mathrm{H}_{2}$ collisions as schematized in the upper right quadrant. The regions where nuclear exchange, dissociation, and nonreactive reactions occur are differentiated. Solid lines correspond to CTDIM calculations with the $V_{\mathrm{GG}}$ (Ref. 27) potential while dashed lines used the $V_{\mathrm{VLA}}$ (Ref. 28) one.

In Fig. 5(d), we qualitatively illustrate NE processes for the case of $b=0.8 a_{0}$ and the corresponding quantitative information is given in Fig. 5(e), where incoming $(\mathrm{C}+\mathrm{AB})$ and outgoing $(\mathrm{B}+\mathrm{AC})$ stages of the collision are separated by a shaded rectangle that indicates the time interval where NE occurs. The large- $R$ attractive part of the potential deviates the projectile $\mathrm{C}$ from its straight-line trajectory towards the internuclear region of $\mathrm{AB}$ where $V_{\mathrm{PES}}$ is highly repulsive [see Fig. 5(e) around $v t=-1 a_{0}$ ]. As $\mathrm{C}$ approaches the closest nucleus (B), it is strongly pushed away (in the longitudinal right direction in our illustration). The strongly decelerated nucleus, $\mathrm{C}$, is then caught by the receding A to finally form the diatom AC with large internal energy [see Fig. 5(e)]. In the case of more distant collisions with $b$ close to $1.5 a_{0}$, the incoming $\mathrm{C}$ does not enter the internuclear $\mathrm{AB}$ region as in Fig. 5(d), but it approaches B, which is therefore ejected in the longitudinal left direction [quite similarly to what happens in Fig. 5(f)]. The remaining nucleus A is weakly affected by the collision dynamics until it is caught by the nucleus $\mathrm{C}$ to form a bound $\mathrm{AC}$ diatom escaping to the right [as $\mathrm{B}$ escaped in Fig. 5(d)]. The kinetic energy of C, after colliding with B, accounts for the (significant) translational energy of the final AC molecule. As for elastic nuclear scattering around $b=2.4 a_{0}$, all energy transfers vanish in the case of NE around $b=1.1$ $a_{0}$ (see Fig. 4); in this case, energy transfers to the final AC diatom are prohibited by the fact that $\mathrm{B}$ experiences a head-on collision with the deflected $\mathrm{C}$ projectile and carries on all the collision energy along its outgoing path.

Concerning nonreactive processes, we display in Fig. 5(f) the trajectories of the three $\mathrm{H}$ nuclei in the $(X, Z)$ plane, emphasizing the $R_{\mathrm{AB}}$ bond. Quantitative information on reaction dynamics is included in the accompanying Fig. 5(g), which shows the temporal evolution of the projectile kinetic energy, the internal and translational diatom energies, and the three-center potential $V_{\mathrm{GG}}$. As the $\mathrm{C}^{+}$projectile approaches to $\mathrm{AB}$, it is accelerated by the large- $R$ attractive part of the potential until it enters the repulsive region of $V_{\mathrm{PES}}$, where the diatom $R_{\mathrm{BC}}$ distance becomes small. C is thus significantly decelerated and the closest target atom, B, strongly pushed towards the other target atom, A. This results in a large internal energy transfer to the $\mathrm{AB}$ diatom and a quite small translational energy transfer [see Fig. 5(e)]. In the case of distant collisions with $b \gg 1.8 a_{0}$, the diatom $R_{\mathrm{BC}}$ distance does not reach small enough values so that the system does not enter the repulsive part of the potential. Therefore, binary $\mathrm{C}+\mathrm{B}$ collisions do not play a major role, and the projectile interacts with both target atoms, $\mathrm{A}$ and $\mathrm{B}$. The final $\mathrm{AB}$ diatom is thus subject to a global motion with significant translational motion and its internal energy is mostly vibrational. Interestingly, all energy transfers vanish in the intermediate $b$-region around $b \sim 2.4 a_{0}$, that would lead to a clamped-nuclei $\mathrm{AB}$ final diatom and an unperturbed $\mathrm{C}$ projectile. This behavior is due to the almost exact balance of the counteracting attractive and repulsive potential effects, also found in the vibrational energy distribution for $b>1.8 a_{0}$ of Ref. 27 .

\section{B. Electron dynamics and inelastic cross sections}

The total SEC cross sections obtained using the $V_{\mathrm{GG}}$ potential are presented in Fig. 6(a), together with the REC [Eq. (8)], DEC [Eq. (5)], and NREC [Eq. (4)] contributions to this cross section. Our results are compared to the experimental ones of Refs. 16-18, recommended data of Ref. 37, and previous theoretical values. . $^{3,4,9,11,21}$

As mentioned in the Introduction, the main difference between our present and previous ${ }^{24}$ CTDIM calculations, which both employ the $V_{\mathrm{GG}}$ potential, is the improved nuclear statistics. In this respect, we show in Table I that increas- 


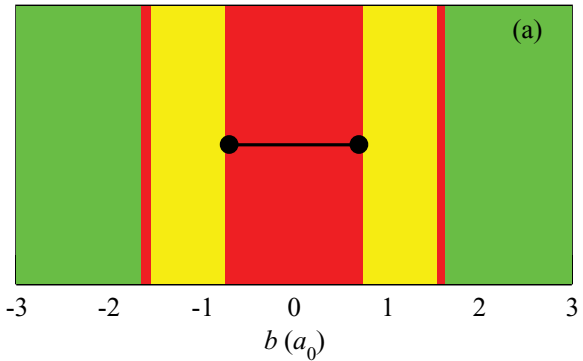

Nuclear exchange

Dissociation

Elastic
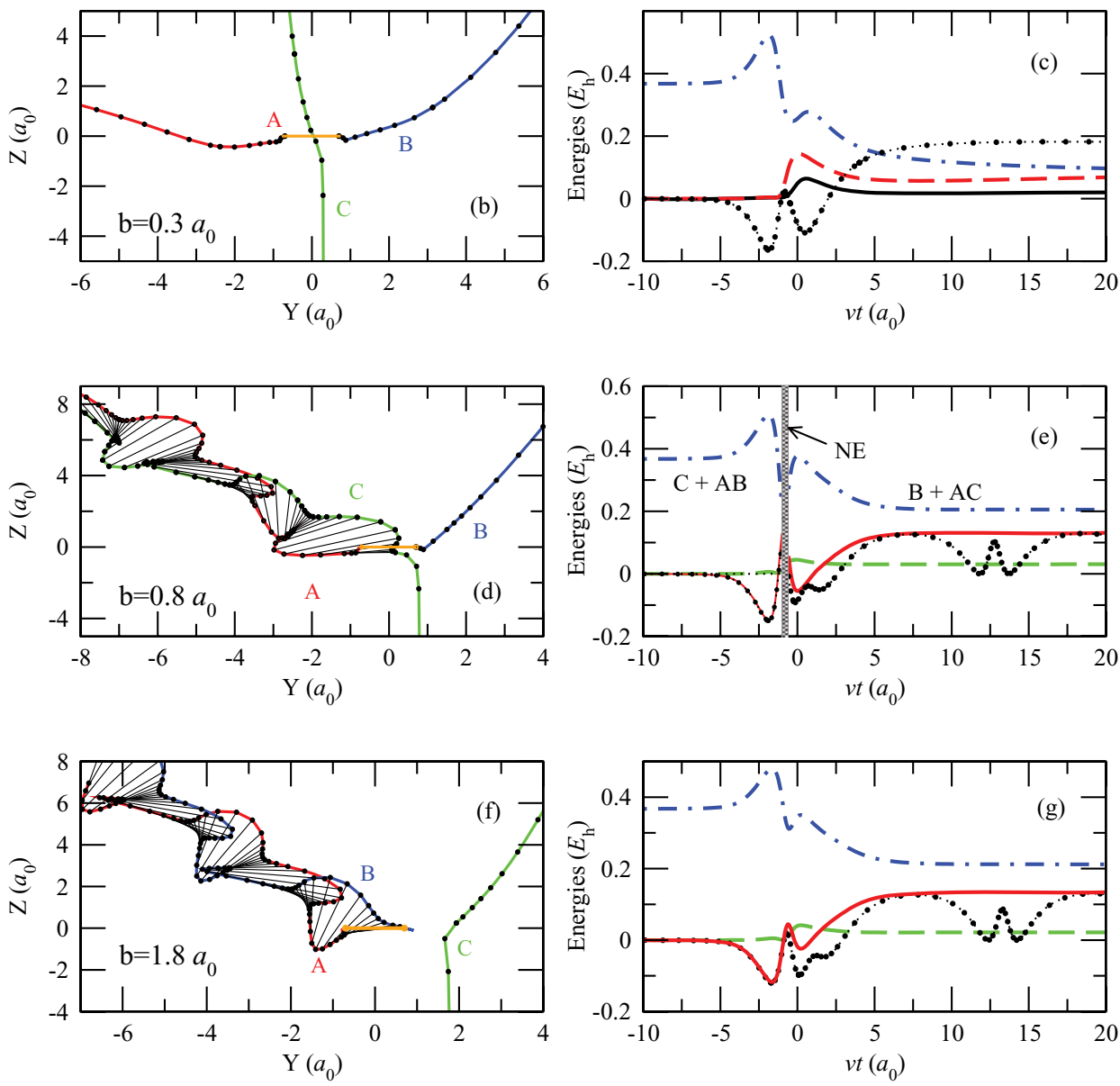

FIG. 5. (a) Impact parameter regions where nuclear exchange, dissociation, and nonreactive reactions occur in $\mathrm{H}^{+}+\mathrm{H}_{2}$ collisions at $E=10$ eV. The initial conditions are the same as in Fig. 4. Panels (b) and (c) illustrate a dissociative trajectory, panels (d) and (e) illustrate a nuclear exchange trajectory, and panels (f) and (g) illustrate a nonreactive trajectory. The impact parameter employed in each pair of panels is indicated in the left-hand-side panel. Panels (b), (d), and (f) illustrate the nuclear motion, while panels (c), (e), and (g) show the temporal evolutions of the projectile kinetic energy (dotted-dashed line), the internal (solid line), and translational (dashed line) diatomic energies, and the $\mathrm{H}_{3}^{+}$potential $V_{\mathrm{GG}}$ (dotted line). $V_{\mathrm{GG}}$ has been shifted so that lim $t \rightarrow-\infty V_{\mathrm{GG}}(t)=0$. In (e), NE occurs around $v t \approx-1 a_{0}$; for $v t<-1 a_{0}$, the projectile is $\mathrm{C}^{+}$while, for $v t>-1 a_{0}$, B is considered as the projectile. In the case of dissociation (c), (solid line), (dashed line) and (dotted-dashed line) correspond to the kinetic energies of the nuclei A, B, and C, respectively.

ing the number of initial trajectories describing the target molecule from 300 to 600 , for each pair of $(v, b)$ values, does not significantly modify the cross sections displayed in Figs. 6 and 7, which can be considered as converged. The present ensemble of 300 initial target geometries allows us to extend the SEC results to lower impact energies than in Ref. 24, so we observe in Fig. 6(a) the rise and fall of the REC cross section defined, according to Eq. (21), as $\sigma_{\mathrm{REC}}^{\mathrm{SEC}}=\sigma_{J=2}^{\mathrm{SEC}}+\sigma_{J=3}^{\mathrm{SEC}}$. The reactive contribution, $\sigma_{\mathrm{REC}}^{\mathrm{SEC}}$, shows a maximum at $E \simeq 6 \mathrm{eV}$. A similar result was obtained in the TSH calculation of Ref. 4, although significant discrepancies exist between the total CTDIM and TSH SEC cross sections. In particular, the TSH total
SEC cross section increases as $E$ increases from 4 to $9 \mathrm{eV}$, and then smoothly decreases for higher $E$ values (up to $30 \mathrm{eV}$ ). Such an energy dependence of the cross section does not agree with the recommended data of Ref. 37, which display a maximum around $6 \mathrm{eV}$. On the other hand, while our CTDIM calculations nicely tend to reproduce the data of Ref. 37 for $E \leq 4$ and $E \geq 8 \mathrm{eV}$, they show a minimum near $6 \mathrm{eV}$. This structure mainly stems from the NREC contribution to the SEC process; the origin of this feature is linked with the inaccuracy of the $V_{\mathrm{GG}}$ PES, in the light of CTDIM calculations performed with the $V_{\mathrm{VLA}}$ potential, shown in Fig. 6(b). In this respect, the IOSA calculations of $\mathrm{Krstic}^{11}$ lead to a 
TABLE I. Cross sections (in units of $10^{-16} \mathrm{~cm}^{2}$ ) for a set of selected collision energies calculated using 300 (values in between brackets) and 600 initial target geometries, for both $V_{\mathrm{VLA}}$ (top half of the table) and $V_{\mathrm{GG}}$ (bottom half of the table) PESs.

\begin{tabular}{lclll}
\hline \hline$E(\mathrm{eV})$ & \multicolumn{1}{c}{$\mathrm{NREC}^{\mathrm{a}}$} & \multicolumn{1}{c}{$\mathrm{REC}^{\mathrm{b}}$} & \multicolumn{1}{c}{$\mathrm{DEC}^{\mathrm{c}}$} & \multicolumn{1}{c}{$\mathrm{VE}^{\mathrm{d}}$} \\
\hline 5 & $0.329(0.322)$ & $0.446(0.452)$ & $1 \times 10^{-5}\left(1 \times 10^{-5}\right)$ & $3.327(3.316)$ \\
20 & $0.677(0.677)$ & $0.124(0.124)$ & $0.396(0.395)$ & $9.609(9.595)$ \\
100 & $0.639(0.639)$ & $0.011(0.012)$ & $0.264(0.263)$ & $8.554(8.489)$ \\
600 & $3.170(3.168)$ & $0.006(0.004)$ & $0.164(0.163)$ & $3.762(3.762)$ \\
5 & $0.267(0.252)$ & $0.314(0.323)$ & $1 \times 10^{-5}\left(1 \times 10^{-5}\right)$ & $4.251(3.062)$ \\
20 & $0.486(0.485)$ & $0.104(0.104)$ & $0.366(0.367)$ & $9.481(9.465)$ \\
100 & $0.763(0.762)$ & $0.015(0.017)$ & $0.175(0.175)$ & $10.654(10.618)$ \\
600 & $3.296(3.293)$ & $1 \times 10^{-5}\left(1 \times 10^{-5}\right)$ & $1 \times 10^{-5}\left(1 \times 10^{-5}\right)$ & $4.800(4.700)$ \\
\hline \hline
\end{tabular}

${ }^{a}$ Nonreactive electron capture [Eq. (4)].

${ }^{\mathrm{b}}$ Reactive electron capture [Eq. (8)].

${ }^{\mathrm{c}}$ Dissociative electron capture [Eq. (5)].

${ }^{\mathrm{d}}$ Vibrational excitation [Eqs. (2), (3) and (7)].

total SEC cross section which exhibits, at $E \approx 6 \mathrm{eV}$, a structure similar to the one found with the $V_{\mathrm{GG}}-\mathrm{CTDIM}$ method. As already mentioned, the nonadiabatic transition leading to SEC takes place when the vibrational energy of the system increases during the collision to become quasi-resonant with the SEC channels, which requires that the trajectory reaches relatively short internuclear distances. As pointed out in Ref. 8, most of these trajectories end up in the product channel, which explains the importance of REC at low energies. It must be

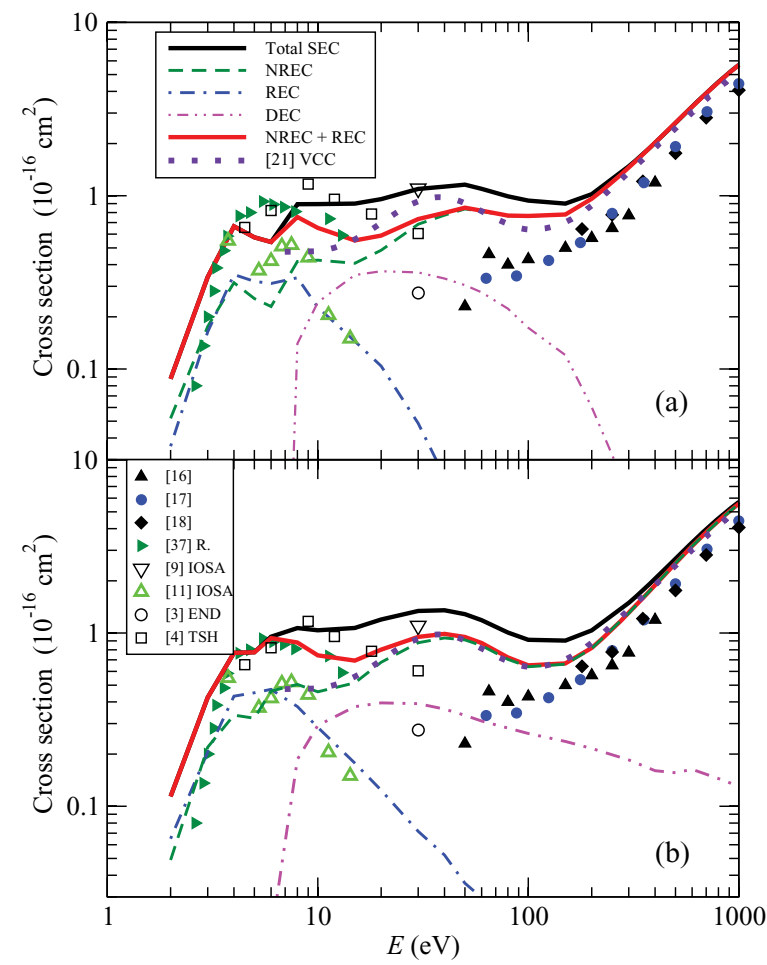

FIG. 6. SEC cross sections, as functions of the impact energy $E$, obtained by means of CTDIM calculations using the $V_{\mathrm{GG}}$ (a) and $V_{\mathrm{VLA}}$ (b) potentials. The contributions of the nonreactive, dissociation, and NE processes to the total SEC cross section are indicated and the results are compared with experimental data for total SEC (solid symbols) (Refs. 16-18), recommended data (Ref. 37), and other calculations (empty symbols) (Refs. 3, 4, 9, 11, 21) as specified in the figure. noted that, although detailed quantum-mechanical treatments have been carried out in the threshold region of the SEC reactions, they have considered isotopically modified species and therefore they are not included in Fig. 6.

In the intermediate impact energy range, $10 \leq E \leq 100$ $\mathrm{eV}$, the shape of our total $V_{\mathrm{GG}}$-CTDIM cross section coincides with that of our VCC results. ${ }^{21}$ Nonetheless, the VCC and CTDIM cross sections differ about $15 \%$, mainly due to the overestimation of the DEC contribution [reaction (5)] to SEC within the CTDIM calculations. If we neglect this contribution, which is much larger than its VCC counterpart over the whole $10 \leq E \leq 100 \mathrm{eV}$ energy range, we indeed obtain a good agreement of the CTDIM SEC with both the VCC total cross section and the recommended data. ${ }^{37}$ The overestimation of DEC cross sections in CTDIM calculations can be a consequence of the nuclear motion being restricted to the fundamental $\mathrm{H}_{3}^{+} \mathrm{PES}$, asymptotically correlated to $\mathrm{H}^{+}+\mathrm{H}_{2}$. This restriction might be responsible for the overestimation of the total SEC cross section with respect to the recommended

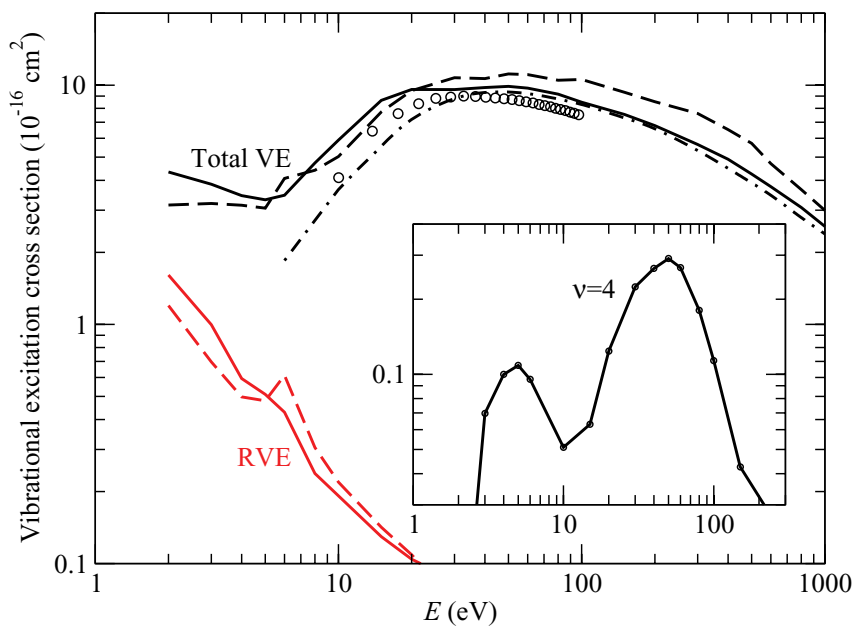

FIG. 7. Total vibrational excitation and RVE cross sections obtained with the CTDIM method and $V_{\mathrm{GG}}$ (dashed line) and $V_{\mathrm{VLA}}$ (solid line) potentials. DECENT (Ref. 30) (dotted-dashed line) and VCC (Ref. 21) calculations (open circles) are also included. The inset shows the $v=4$ partial vibrational excitation cross section from $V_{\mathrm{GG}}$-CTDIM calculations. 
data in the threshold region. A possible improvement would be to introduce (at least) one excited $\mathrm{H}_{3}^{+} \mathrm{PES}$, correlated to asymptotic $\mathrm{H}+\mathrm{H}_{2}^{+}$channels, to correctly resolve the vibrational distribution within the SEC process.

For $E>100 \mathrm{eV}$, only non-rearrangement paths contribute to the nonadiabatic processes. The $V_{\mathrm{GG}}-\mathrm{CTDIM}$ and VCC total SEC cross sections are here in very good agreement. Both of them lie $\sim 20 \%$ higher than the experimental data of Refs. 16 and 17. The reliability of the computed cross sections is ascertained by the fact that they adequately tend to the well-established vibrational sudden and FC limits, which are accurate for $E \geq 300 \mathrm{eV}$ and $1000 \mathrm{eV}$, respectively (see Ref. 15).

In Fig. 6(b), the CTDIM calculations have been performed using $V_{\mathrm{VLA}}$ for the nuclear motion. As can be seen in this figure, the $V_{\mathrm{VLA}}$-CTDIM results show better agreement with the experiments than those using $V_{\mathrm{GG}}$, in both low and intermediate impact energy ranges. This is particularly true if one neglects the DEC contribution to SEC, which is overestimated because of the main shortcoming of the CTDIM approach previously discussed. The sum of REC and NREC cross sections is in striking agreement with both the recommended data, based on experimental measurements of Ref. 37 for $E \leq 10 \mathrm{eV}$, and our VCC results for $E>10 \mathrm{eV}$. The $V_{\mathrm{VLA}}-\mathrm{CTDIM}$ calculation does not yield the spurious minimum of the total SEC cross section at $E \approx 6 \mathrm{eV}$, obtained with $V_{\mathrm{GG}}$ [Fig. 6(a)]; furthermore, in the $V_{\mathrm{VLA}}$-CTDIM calculation, the oscillating structure of the NREC cross section, combined with the bell-shaped REC contribution, leads to a small kink in the total SEC cross section that can also be distinguished in the recommended data ${ }^{37}$ at $E \approx 5 \mathrm{eV}$.

For $E>10 \mathrm{eV}$, the NREC $\sigma_{J=1}^{\mathrm{SEC}}$ cross section obtained with $V_{\mathrm{VLA}}$-CTDIM method coincides almost exactly with the total VCC one. From a formal point of view, the VCC calculations, based on IOSA, should give total SEC cross sections that include nuclear rearrangement process, if the calculation were performed in an infinite basis of the nonreactive space. ${ }^{11,38}$ The agreement between NREC, $\sigma_{J=1}^{\mathrm{SEC}}$, and $\sigma_{\mathrm{VCC}}^{\mathrm{SEC}}$, at $E>10 \mathrm{eV}$, indicates that $\mathrm{VCC}$ does not implicitly account for REC processes. The same applies to the IOSA results of Ref. 11, which disagree with both the experimental data and other theoretical results. We are thus led to conclude that it is essential to explicitly include nuclear exchange paths for $E \leq 20 \mathrm{eV}$.

For $10 \leq E \leq 300 \mathrm{eV}$, SEC occurs through a twostep mechanism involving intermediate excitation states $\mathrm{H}^{+}+\mathrm{H}_{2}(v=3,4) .{ }^{20}$ The same mechanism still tailors the NREC process at $E<10 \mathrm{eV}$. As a matter of fact, the $\mathrm{H}^{+}+\mathrm{H}_{2}(v \geq 3)$ vibrational cross sections present the same two-humped camel shape as the total SEC cross section, as shown in the inset of Fig. 7 for $v=4$. Intermediate excitation states with $v>3$ are also involved in REC process. As a result, both high-lying $v$ vibrational and SEC cross sections show a maximum about $E \sim 5$ and $30 \mathrm{eV}$. In both cases, the first maximum, stems from equivalent contributions of REC and NREC reactions while the outer one is only due to the latter one.

We now turn our attention to the VE cross sections. We present in Fig. 7 the total cross section, $\sigma^{\mathrm{VE}}$, and its RVE contribution, $\sigma_{J=2}^{\mathrm{VE}}+\sigma_{J=3}^{\mathrm{VE}}$, as functions of $E$. We distinguish the results obtained from CTDIM calculations employing $V_{\mathrm{GG}}$ and $V_{\mathrm{VLA}}$. The contribution of the dissociative process (reaction (3)) is not displayed since it is negligible over the whole impact energy range. The shape of our $V_{\mathrm{GG}}$-CTDIM total VE cross section agrees with that of the result of Ref. 30 at $6<E<1000 \mathrm{eV}$, but lies $\sim 20 \%$ above it. This discrepancy cannot be related to the fact that $\mathrm{NE}$ and dissociative nuclear trajectories were discarded in DECENT calculations, ${ }^{30}$ since the contribution of such trajectories to the VE process is very small for $E>6 \mathrm{eV}$. The fact that the electron dynamics is not explicitly taken into account in the DECENT model is not relevant, since $c_{J=1}(b) \sim 1$ for those large $b$ 's which mostly contribute to the VE cross section at intermediate and high impact energies. The discrepancy rather stems from the fact that our CTDIM approach employs a statistical procedure among nuclear trajectories to compute the cross sections [see Eq. (23)] while the DECENT model assumes an initially frozen $\mathrm{H}_{2}$ target and a Poisson probability distribution among the final vibrational states, which is only formally exact in the case of a harmonic oscillator perturbed by an external potential, linear in the vibrational coordinate. In view of the differences between these two approaches, the $\sim 20 \%$ discrepancy is small.

The maximum of the total VE cross section obtained with $V_{\mathrm{VLA}}$-CTDIM is shifted to lower $E(\approx 30 \mathrm{eV})$ with respect to the corresponding $V_{\mathrm{GG}}$-CTDIM one. Beyond this maximum, the $V_{\mathrm{VLA}}$-CTDIM total VE cross section lies close to the DECENT results. Interestingly, RVE is sizable for $E \leq 10$ $\mathrm{eV}$ and increases as $E$ decreases down to $2 \mathrm{eV}$, where $\left(\sigma_{J=2}^{\mathrm{VE}}\right.$ $\left.+\sigma_{J=3}^{\mathrm{VE}}\right) / \sigma_{\text {total }}^{\mathrm{VE}} \sim 50 \%$. As mentioned before for the SEC process, the VCC calculations do not implicitly includes RVE channels, which explains the faster decrease than its $V_{\mathrm{VLA}^{-}}$ CTDIM counterpart as $E$ decreases for $10<E<20 \mathrm{eV}$.

While the $V_{\mathrm{VLA}}-\mathrm{CTDIM}$ RVE cross section smoothly decreases as $E$ increases, a bump appears in its $V_{\mathrm{GG}}$-CTDIM counterpart at $E=6 \mathrm{eV}$. This structure compensates the artificial minimum observed in the NREC cross section in Fig. 6(a). This emphasizes that small inaccuracies in the PES, as observed in Fig. 3, strongly influence the nuclear dynamics and the subsequent electron dynamics.

\section{Electron dynamics along NE paths and around conical intersections}

To illustrate the interplay between the nuclear exchange and electron transfer mechanisms within REC processes, we consider the initial conditions of Fig. 5(d), which correspond to proton, with $E=10 \mathrm{eV}$ and $b=0.8 a_{0}$, impinging on a, initially frozen, $\mathrm{H}_{2}$ molecule along the relative projectiletarget orientation TII (see Fig. 1). The REC reaction is then $\mathrm{C}^{+}+\mathrm{AB} \rightarrow \mathrm{B}+\mathrm{AC}^{+}$. Figure 8(a) displays the temporal evolutions of the diatomic distances $R_{\mathrm{AB}}, R_{\mathrm{AC}}$, and $R_{\mathrm{BC}}$ as functions of the scaled time $v t$. In Fig. 8(b), we jointly report $\left|1-c_{1}(t)\right|^{2}$ and $\left|1-c_{2}(t)\right|^{2}$, which correspond, at time $t$, to the probabilities for SEC leading to $\mathrm{C}+\mathrm{AB}^{+}$and $\mathrm{B}+\mathrm{AC}^{+}$, respectively. The probability to form $\mathrm{AB}^{+}$starts to differ from 0 quite early in the incoming part of the collision, $v t \approx-7 a_{0}$, where $R_{\mathrm{AC}} \approx R_{\mathrm{BC}} \approx 7 a_{0}$. Around $v t \approx-1 a_{0}$, the nucleus $\mathrm{C}$ has reached the distance of closest approach to 

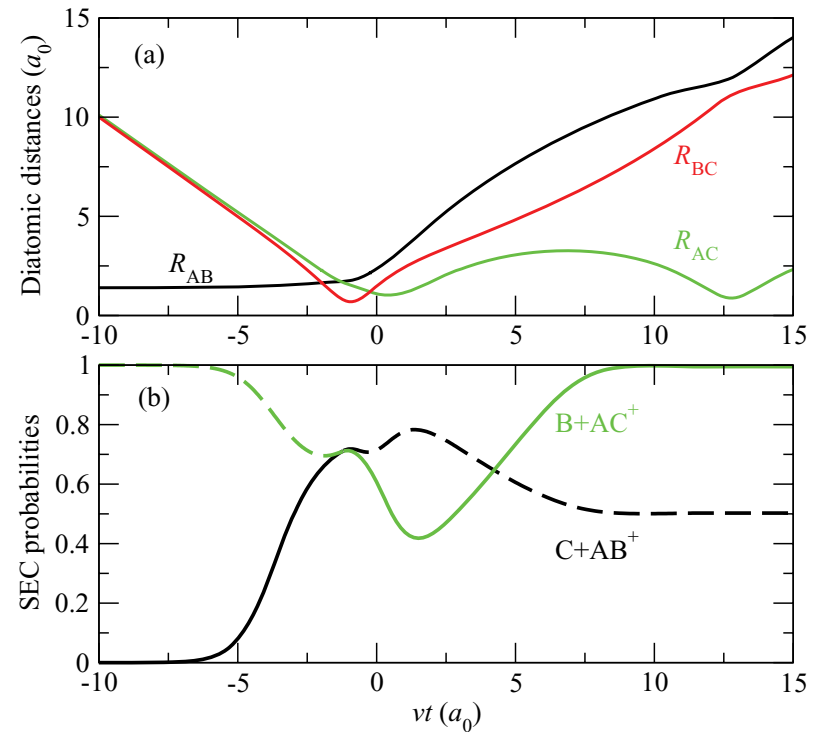

FIG. 8. Temporal evolution of the diatomic distances (a) and SEC probabilities for the formation of $\mathrm{C}+\mathrm{AB}^{+}$and $\mathrm{B}+\mathrm{AC}^{+}(\mathrm{b})$, in $\mathrm{C}^{+}+\mathrm{AB}$ collisions with $b=0.8 a_{0}$ and $E=10 \mathrm{eV}$, as functions of the scaled time $v t$. The target is initially at equilibrium with zero vibrational energy, and the relative projectile-target orientation is TII (see Fig. 1). In panel (b), NE occurs at $v t \approx-1 a_{0}$; we report the probabilities using dashed lines when the threecenter system is not in the corresponding arrangement.

nucleus $\mathrm{B}\left(R_{\mathrm{BC}} \sim 1 a_{0}\right)$ and $\left|1-c_{1}(t)\right|^{2} \sim 0.7$; $\mathrm{C}$ has been strongly decelerated [see Fig. 5(e)] and NE is going to occur through ejection of $\mathrm{B}$ and repulsion on A [see Fig. 5(d)]. At the same moment, $\left|1-c_{1}\right|^{2}=\left|1-c_{2}\right|^{2}$, so that the $\mathrm{C}+\mathrm{AB}^{+}$ and $\mathrm{B}+\mathrm{AC}^{+} \mathrm{SEC}$ probabilities can be smoothly joined at $v t \sim-1 a_{0}$ in Fig. 8(b). Clearly, NE and electron transfer appear as simultaneous processes.

Another characteristic feature of the electron dynamics at low energy $\mathrm{H}^{+}+\mathrm{H}_{2}$ collisions that deserves particular attention is the importance of SEC transitions that take place near the conical intersection (CI) between the two lowest $\mathrm{H}_{3}^{+}$PESs as $\rho=\rho_{c}=2.5 a_{0}$ and large $R$. Suárez et al. ${ }^{31}$ have recently shown with a semiclassical model that, beyond moleculartype transitions, asymptotic transitions at CIs can indeed be significant. Our present calculations support this conclusion. To illustrate this point, we consider the case of NREC after a $\mathrm{C}^{+}+\mathrm{AB}$ collision with $E=5 \mathrm{eV}, b=0.8 a_{0}$, and initial target conditions $\rho=\rho_{0}$ and $E_{\text {vib }}=0$. Figure 9 presents the temporal evolution of the SEC probability, $P_{\mathrm{SEC}}$, and the diatomic $\rho$ and projectile $R$ distances. An important SEC transition takes place at $v t \approx-5 a_{0}$ as $\mathrm{C}^{+}$enters into the molecular (three-center) region $\left(R \leq 5 a_{0}\right)$. Nevertheless, the projectile leaves this region in the outgoing phase of the collision with $P_{\mathrm{SEC}} \sim 0$ because of a symmetric transition at $v t \approx 5 a_{0}$. We thus have an excited $\mathrm{AB}$ molecule that strongly vibrates and whose diatomic distance $\rho$ regularly crosses the $\rho_{c}$ value. For $v t>8 a_{0}$, all noticeable variations of $P_{\mathrm{SEC}}$ correspond to the system crossing the CI (about $v t=10,13$, 17, 20, and $23 a_{0}$ in Fig. 9). Later on, $R$ is very large ( $v t>25$ $a_{0}$ ), the CI becomes strongly peaked and it is traversed diabatically. The consequence is that $P_{\mathrm{SEC}} \neq 0$ as $t \rightarrow \infty$ because of asymptotic (large- $R$ ) transitions around the CI. Beyond the specific case examined in Fig. 8, the role of

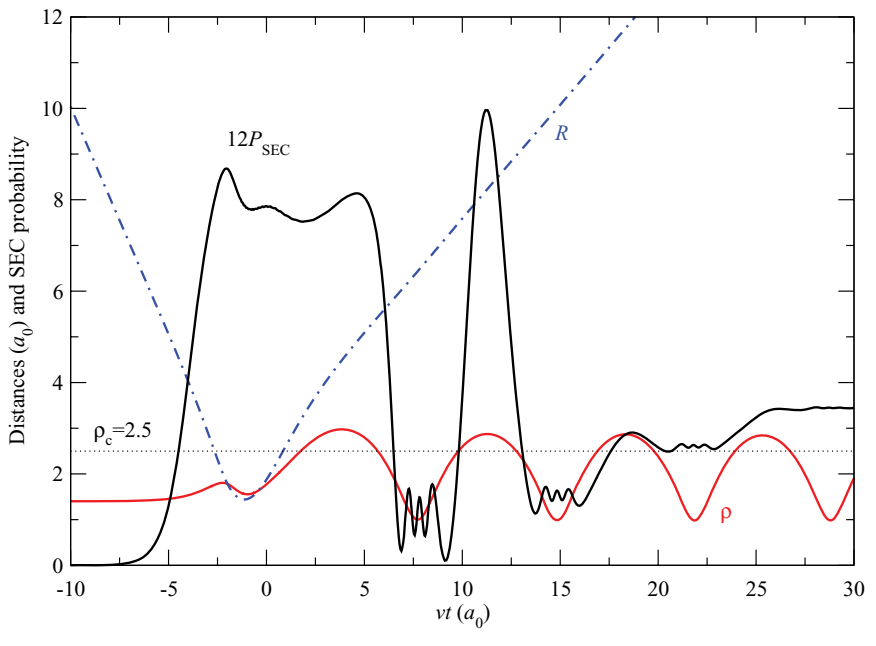

FIG. 9. Temporal evolutions, as functions of the scaled time $v t$, of the SEC probability, diatomic distance $\rho$, and projectile-target distance $R$, in $5 \mathrm{eV}$ $\mathrm{H}^{+}+\mathrm{H}_{2}$ collisions with $b=0.8 a_{0}$. The target is initially at equilibrium with zero vibrational energy, and the relative projectile-target orientation is TI (see Fig. 1). The SEC probability has been multiplied by 12 .

CI-localized transitions generally becomes less important as $E$ increases, and molecular radial transitions take over.

\section{CONCLUSIONS AND PERSPECTIVES}

$\mathrm{H}^{+}+\mathrm{H}_{2}$ collisions have been studied in a wide range of impact energies $(E=2-1000 \mathrm{eV})$ by means of the semiclassical CTDIM method, which employs Monte Carlo statistics and classical mechanics to describe the nuclear motion, and the DIM approach to describe (quantum mechanically) electron transitions. We have improved our previous study $^{24}$ by employing a larger (and converged) nuclear statistics, and investigated the effect of the accuracy of the $\mathrm{H}_{3}^{+}$ PES parametrization on dynamical results by using the pioneering parametrization of Giese and Gentry, ${ }^{27} V_{\mathrm{GG}}$, and the most recent one of Velilla et al., ${ }^{28} V_{\mathrm{VLA}}$. We have found that inelastic cross sections are indeed sensitive to the accuracy of the PES from low (few eV) to intermediate $(100 \mathrm{eV})$ impact energies. The differences are relatively large at low $E(\sim 5 \mathrm{eV})$, where some small deviations in the PES lead to spurious structures in the cross sections. Given the accuracy of the PES parametrization of Ref. 28, further improvement of the ground state PES is not expected to significantly modify the dynamical results at $E>2 \mathrm{eV}$ with the present method.

$V_{\mathrm{VLA}}$-CTDIM calculations yield a total SEC cross sections that fairly agrees with measurements at low $E$ and reproduces the shape of our previous vibronic calculations ${ }^{21}$ in the intermediate energy range, where there is a lack of experimental data and independent calculations are, therefore, needed. The agreement with both the recommended data at low $E$ and with the VCC results is very good if one neglects the DEC [Eq. (5)] contribution to the total cross section. The sum of NREC [Eq. (4)] and REC [Eq. (8)] cross sections, that we can assimilate to the recommended theoretical data for the total SEC, produces a double-humped shape with 
maxima located at $E \sim 6 \mathrm{eV}$ and $E \sim 30 \mathrm{eV}$. The first maximum corresponds to energies where nuclear exchange paths are of paramount importance, while the second one is due to nonreactive paths. In both energy regions, SEC from $\mathrm{H}_{2}(v$ $=0$ ) occurs through a two-step mechanism involving intermediate excitation $\mathrm{H}^{+}+\mathrm{H}_{2}(v)$ states; NREC implies quasiresonant $v=3,4$ states and reactive paths involve even more excited vibrational states. Besides, the agreement at high energies between our cross sections and those from close-coupling calculations in terms of $a b$ initio wavefunctions supports the usefulness of the DIM treatment.

The semiclassical nature of the CTDIM approach has further allowed us to picture the main features of nuclear and electron dynamics in $\mathrm{H}^{+}+\mathrm{H}_{2}$ collisions. At large impact parameters, where all of the nuclear paths are nonreactive, nuclear energy is preferably converted into translational energy of the final diatom, while short impact parameter collisions favor energy transfer to the internal degrees of freedom of the diatom. We have found that nuclear exchange and electron transitions are simultaneous processes, notwithstanding the fact that significant asymptotic electron capture transitions can take place at low $E$ around conical intersections, as predicted in Ref. 31.

The shortcoming of the present CTDIM method is the use of only the ground PES to drive the nuclear motion. The comparison with vibronic calculations indicates that this drawback leads to an overestimation of the DEC [Eq. (5)] cross section at intermediate $(10-100 \mathrm{eV})$ energies. Given that CTDIM decouples nuclear and electron dynamics, it is not feasible to overcome or circumvent the problem in a pure CTDIM framework, and it is difficult to gauge the influence of this approximation at $E<4 \mathrm{eV}$. Nonetheless, the present work can be used as a basis to implement a hemiquantal treatment of $\mathrm{H}^{+}+\mathrm{H}_{2}$ collisions, in which the nuclear motion is classically tailored by a dynamical common PES, which is selfconsistently built according to the quantum electron dynamics (see, for instance, Refs. 39 and 40). This work is under progress. On the other hand, the electronic part of the calculation could be improved by considering a more accurate set of diabatic surfaces for the ground and excited states, such as those of Ref. 41.

\section{ACKNOWLEDGMENTS}

This work has been partially supported by the DGICYT project ENE2007-62934.
${ }^{1}$ S. P. Oh and Z. Haiman, Astrophys. J. 569, 558 (2002).

${ }^{2}$ D. W. Savin, P. S. Krstić, Z. Haiman, and P. C. Stancil, Astrophys. J. 606, L167 (2004)

${ }^{3}$ J. Morales, A. Diz, E. Deumens, and Y. Öhrn, J. Chem. Phys. 103, 9968 (1995).

${ }^{4}$ A. Ichihara, O. Iwamoto, and R. Janev, J. Phys. B 33, 4747 (2000).

${ }^{5}$ H. Kamisaka, W. Bian, K. Nobosuda, and H. Nakamura, J. Chem. Phys. 116, 654 (2002).

${ }^{6}$ T. S. Chu and K. L. Han, J. Phys. Chem A 109, 2050 (2005).

${ }^{7}$ R. F. Lu, T. S. Chu, and K. L. Han, J. Phys. Chem A 109, 6683 (2005).

${ }^{8}$ B. Li and K. L. Han, J. Chem. Phys. 128, 11416 (2008).

${ }^{9}$ M. Baer, G. Niedner-Schatteburg, and J. P. Toennies, J. Chem. Phys. 91, 4169 (1989).

${ }^{10}$ A. Saieswari and S. Kumar, Chem. Phys. Lett. 449, 358 (2007).

${ }^{11}$ P. S. Krstić, Phys. Rev. A 66, 042717 (2002).

${ }^{12}$ P. S. Krstić and R. K. Janev, Phys. Rev. A 67, 022708 (2003).

${ }^{13}$ S. Amaran and S. Kumar, J. Chem. Phys. 128, 064301 (2008).

${ }^{14}$ C. Illescas and A. Riera, J. Phys. B 31, 2777 (1998).

${ }^{15}$ D. Elizaga, L. F. Errea, J. D. Gorfinkiel, A. Macías, L. Méndez, A. Riera, and A. Rojas, J. Phys. B 33, 2037 (2000).

${ }^{16}$ W. H. Cramer, J. Chem. Phys. 35, 836 (1961).

${ }^{17}$ T. Kusakabe, L. Pichi, R. J. Buenker, M. Kimura, and H. Tawara, Phys. Rev. A 70, 052710 (2004).

${ }^{18}$ M. W. Gealy and B. I. Van Zyl, Phys. Rev. A 36, 3091 (1987).

${ }^{19}$ L. F. Errea, A. Macías, L. Méndez, I. Rabadán, and A. Riera, Phys. Rev. A 65, 010701(R) (2001).

${ }^{20}$ G. Niedner, M. Noll, J. P. Toennies, and C. Schlier, J. Chem. Phys. 87, 2685 (1987).

${ }^{21}$ L. F. Errea, L. Fernández, L. Méndez, B. Pons, I. Rabadán, and A. Riera, Phys. Rev. A 75, 032703 (2007).

${ }^{22}$ F. O. Ellison, J. Am. Chem. Soc. 85, 3540 (1963).

${ }^{23}$ A. V. Phelps, J. Phys. Chem. Ref. Data 19, 653 (1990).

${ }^{24}$ L. F. Errea, C. Illescas, A. Macías, L. Méndez, B. Pons, I. Rabadán, and A. Riera, J. Phys. B 42, 105207 (2009).

${ }^{25}$ R. Abrines and I. C. Percival, Proc. Phys. Soc. London 88, 861 (1966).

${ }^{26}$ R. Abrines and I. C. Percival, Proc. Phys. Soc. London 88, 873 (1966).

${ }^{27}$ C. F. Giese and W. R. Gentry, Phys. Rev. A 10, 2156 (1974).

${ }^{28}$ L. Velilla, B. Lepetit, A. Aguado, J. A. Beswick, and M. Paniagua, J. Chem. Phys. 129, 084307 (2008).

${ }^{29}$ L. F. Errea, J. D. Gorfinkiel, A. Macías, L. Méndez, and A. Riera, J. Phys. B 30, 3855 (1997).

${ }^{30}$ W. R. Gentry and C. F. Giese, Phys. Rev. A 11, 90 (1975).

${ }^{31}$ J. Suárez, L. F. Errea, C. Illescas, A. Macías, L. Méndez, B. Pons, I. Rabadán, and A. Riera, Phys. Rev. A 77, 012708 (2008).

${ }^{32}$ E. P. Wigner, Phys. Rev. 40, 749 (1932).

${ }^{33}$ N. G. D. Eichenauer and W. Scheid, J. Phys. B 14, 3929 (1981).

${ }^{34}$ P. Botheron and B. Pons, Phys. Rev. A 80, 023402 (2009).

${ }^{35}$ P. Barragán, L. F. Errea, A. Macías, L. Méndez, I. Rabadán, and A. Riera, J. Chem. Phys. 124, 184303 (2006).

${ }^{36}$ M. Baer, Phys. Rep. 358, 71 (2002).

${ }^{37}$ F. Linder, R. K. Janev, and J. Botero, in Atomic and Molecular Procceses in Fusion Edge Plasmas, edited by R. K. Janev (Plenum, New York, 1995).

${ }^{38} \mathrm{~K}$. T. Tang, in Theory of Chemical Reaction Dynamics II, edited by M. Baer (CRC, Boca Raton, FL, 1985).

${ }^{39}$ D. J. Diestler, J. Chem. Phys. 78, 2240 (1983).

${ }^{40}$ L. L. Halcomb and D. J. Diestler, J. Chem. Phys. 84, 3130 (1986).

${ }^{41}$ L. P. Viegas, A. Alijah, and A. J. C. Varandas, J. Chem. Phys. 126, 074309 (2007) 